\title{
Extending the scope of microscopic solvability: Combination of the Kruskal-Segur method with Zauderer decomposition
}

\author{
Thomas Fischaleck and Klaus Kassner \\ Institut für Theoretische Physik, Otto-von-Guericke-Universität Magdeburg, \\ Postfach 4120, D-39016 Magdeburg, Germany
}

PACS 47.54.-r - Pattern selection; pattern formation

PACS 11.10.Jj - Asymptotic problems and properties

PACS 81.10.Aj - Theory and models of crystal growth

PACS 68.70.+w - Whiskers and dendrites (growth, structure, and nonelectronic properties)

\begin{abstract}
Successful applications of the Kruskal-Segur approach to interfacial pattern formation have remained limited due to the necessity of an integral formulation of the problem. This excludes nonlinear bulk equations, rendering convection intractable. Combining the method with Zauderer's asymptotic decomposition scheme, we are able to strongly extend its scope of applicability and solve selection problems based on free boundary formulations in terms of partial differential equations alone. To demonstrate the technique, we give the first analytic solution of the problem of velocity selection for dendritic growth in a forced potential flow.
\end{abstract}

The fundamental equations describing the growth of a crystal into its undercooled melt are very difficult to solve, if surface tension effects are accounted for, even when re' striced to the simplest case of merely diffusive heat transport. On the other hand, the capillary length $d_{0}$ describing these effects is typically very small in comparison with ' other length scales of the problem such as the sizes of growing patterns or the diffusion length. Therefore, it was a natural step to first look for solutions with $d_{0}$ set equal to zero. This simplified problem was solved exactly by Ivantsov [1] who showed that the crystal can grow in the shape of a parabola in $2 \mathrm{D}$ or a paraboloid in 3D. A major drawback of these solutions is that they constitute a whole continuum for any given undercooling: the mathematics fixes only the Péclet number $P_{c}=V \rho / D$, where $V$ is the growth velocity of the crystal, $\rho$ the tip radius of the parabolic needle, and $D$ the thermal diffusion coefficient. Hence, only the product of velocity and length scale is determined, but neither of the two quantities separately. In experiments, a given undercooling leads to both a welldefined growth velocity and a well-defined tip radius of the needle crystal, which after developing side branches is called a dendrite. This situation became known as the $s e-$ lection problem of diffusion-limited dendritic growth and is was not solved until some twenty years ago [2-5], with the advent of microscopic solvability theory.

Because the theory was mathematically complex and not very intuitive, it failed to enjoy unanimous appraisal. Moreover, its success in explaining experiments remained controversial to some extent [6]. It has been emphasized by Tanveer [7] that even small fluid flows in the melt might account for changes in the theoretically predicted scalings as the problem is structurally unstable. Hence, selection theory should be extended to nondiffusive transport such as convection. To our knowledge, the only approach to solvability theory available so far for models with convection is due to Bouissou and Pelcé (BP) [8]. Their method relies on a linearized solvability condition, which prevents it from becoming exact in the limit of vanishing $d_{0}$. Also, it has been shown [9] that nonlinearity may be crucial in problems involving multiple parameters. Hence, a method would be more than desirable that takes nonlinear solvability into account but can also deal with problems not permitting an integral formulation. This letter presents such an approach.

Lengths are nondimensionalized by the tip radius $\rho$ of the Ivantsov parabola [1], which is then given by $y-y_{0}=$ $\frac{1}{2}-\frac{1}{2} x^{2}$ in a comoving coordinate system. We use conformal parabolic coordinates $x=\xi \eta, y-y_{0}=\frac{1}{2}\left(\eta^{2}-\xi^{2}\right)$, so the Ivantsov parabola reads $\eta=1$. The relevant nondimensional parameters of the problem are the growth Péclet number $P_{c}$, defined above, the stability parameter $\sigma$, and the flow Péclet number $P_{f}$. The latter are given in terms of physical quantities by $\sigma=2 d_{0} D /\left[V \rho^{2}\right]$, 
$P_{f}=U \rho / D$, where $U$ is the velocity of an imposed flow.

In order to demonstrate the power of the method, we first show how it simplifies a solved problem, the growth of of a needle crystal under purely diffusive heat transport. Next we deal with the hitherto unsolved selection problem of a crystal growing in a potential flow, where the basic field equations are nonlinear. We simplify the presentation using some approximations that can in principle be avoided and will be removed in a more extended version of this article. The first problem was treated via the KruskalSegur method [10] by Ben Amar et al. [3]. They start from an integral equation describing steady state growth in the limit $P_{c} \rightarrow 0$. The integral term is linearized about the Ivantsov parabola, setting $\eta_{s}=1+h\left[\eta_{s}(\xi)\right.$ is the interface position]. After reducing the integral to a local expression using sophisticated complex analysis [5], one finds the dominant behavior of the solution near a singularity in the complex plane at $\xi=-i$ :

$$
\sigma \kappa=(1-i \xi) h(\xi),
$$

where

$$
\begin{aligned}
\kappa=\frac{-1}{\left(\xi^{2}+(1+h)^{2}\right)^{\frac{1}{2}}} & \left\{\frac{h^{\prime \prime}}{\left(1+h^{\prime 2}\right)^{\frac{3}{2}}}\right. \\
& \left.+\frac{\xi h^{\prime}-1-h}{\left(\xi^{2}+(1+h)^{2}\right)\left(1+h^{\prime 2}\right)^{\frac{1}{2}}}\right\}
\end{aligned}
$$

is the curvature the prime denotes a derivative w.r.t. the argument (i.e. $\xi$ )]. Equation (1), a second-order nonlinear differential equation for $h(\xi)$, contains all the information needed to compute the transcendental corrections (i.e., the mismatch function) that have to be suppressed at the needle tip for selection to be possible. Essential for its derivation was the use of an integral equation, available only for linear bulk equations.

We now rederive Eq. (11) from the differential equation formulation of the free-boundary problem directly. The field equation for the problem linearized about the Ivantsov solution is just the Laplace equation $\partial_{\xi}^{2} T+\partial_{\eta}^{2} T=$ 0 , both in the liquid and solid phases. The interface boundary conditions become:

$$
\begin{aligned}
\left.T\right|_{s}=-\frac{\sigma}{2} \kappa,\left.\quad T\right|_{l} & =\left.T\right|_{s}+h, \\
h+\xi h^{\prime}+\left(\partial_{\eta}-h^{\prime} \partial_{\xi}\right)\left(\left.T\right|_{l}-\left.T\right|_{s}\right) & =0 .
\end{aligned}
$$

Of these, the first equation is the Gibbs-Thomson relation, the second expresses continuity of the temperature at the interface (subscripts $l$ and $s$ refer to evaluation at the position of the Ivantsov parabola), the third is the continuity equation. Noting that $\left(\partial_{\xi}^{2}+\partial_{\eta}^{2}\right)=\left(\partial_{\xi}+i \partial_{\eta}\right)\left(\partial_{\xi}-i \partial_{\eta}\right)$, we replace the bulk equation with

$$
\begin{array}{lll}
\left(\partial_{\xi}+i \partial_{\eta}\right) T & =0 & \text { in the liquid }, \\
\left(\partial_{\xi}-i \partial_{\eta}\right) T=0 & \text { in the solid. }
\end{array}
$$

From Eqs. (3), (41) and (5) we get, neglecting terms quadratic in $h^{\prime}$ [11]

$$
[(1-i \xi) h]^{\prime}=-2\left(\left.T\right|_{s}\right)^{\prime}=\sigma \kappa^{\prime},
$$

and after one integration, obtaining the integration constant from the boundary condition $h \rightarrow 0(\xi \rightarrow \infty)$, we recover (11) almost effortless.

Let us briefly discuss the philosophy of this approach. The temperature field satisfies the Laplace equation, solved by $T(\xi, \eta)=f_{1}[\xi+i(\eta-1)]+f_{2}[\xi-i(\eta-1)]$. After inserting this general solution into the boundary conditions (3), valid at $\eta=1$, we analytically continue these to the vicinity of $w=-i$ ( $w$ is the analytic continuation of $\xi$ ). Some of the terms must become singular there to compensate for the singularity of the curvature term. Since the solution for the liquid must be analytic in the upper half plane $(\eta>1)$, the $f_{2}$ term remains regular near $w=-i$ and is hence negligible. The other term is a solution of (41). Similarly, dropping regular terms from the solid-side solution, we keep the term that solves (5). This procedure gives a valid approximation near the singularity. Far away from singularities it is also justified, because the curvature term in Eqs. (3) can be linearized and the corresponding inhomogeneity ignored. We also have to take care of the singularity at $w=i$. Since the final result has to be real, this singularity leads to the complex conjugate.

Let us determine the transcendental mismatch for later reference when considering the case with flow. Far from the singularity, the homogeneous part of the linearized Eq. (11) reads

$$
\sigma\left(h^{\prime \prime}+\frac{\xi}{1+\xi^{2}} h^{\prime}\right)+\sqrt{1+\xi^{2}}(1-i \xi) h=0 .
$$

This may be solved using WKB techniques, yielding an outer WKB solution:

$$
\begin{aligned}
h & =B(1-i \xi)^{-\frac{5}{8}}(1+i \xi)^{-\frac{3}{8}} \exp \left\{\sigma^{-\frac{1}{2}} S(\xi)\right\}, \\
S(\xi) & =i \int_{-i}^{\xi}\left(1-i \xi^{\prime}\right)^{\frac{3}{4}}\left(1+i \xi^{\prime}\right)^{\frac{1}{4}} \mathrm{~d} \xi^{\prime} .
\end{aligned}
$$

To obtain the inner equation near $\xi=-i$, we rescale

$$
h=\sigma^{\alpha} \phi, \quad \xi=-i+i \sigma^{\alpha} t .
$$

From equation (11), we have

$$
\sigma^{2 \alpha} t \phi=\sigma^{1-\frac{3}{2} \alpha} K+O\left(\sigma^{-\frac{1}{2} \alpha+1}\right),
$$

where

$$
K=\frac{1}{(2 t+2 \phi)^{\frac{1}{2}}}\left\{\frac{\ddot{\phi}}{\left(1-\dot{\phi}^{2}\right)^{\frac{3}{2}}}+\frac{\dot{\phi}+1}{(2 t+2 \phi)\left(1-\dot{\phi}^{2}\right)^{\frac{1}{2}}}\right\} .
$$

To balance both sides of equation (11), we need $\alpha=\frac{2}{7}$, hence $t \phi=K$, and the asymptotic behavior of $\phi$ for large $t$ is $\phi \sim 2^{-\frac{3}{2}} t^{-\frac{5}{2}}$. Linearizing about the asymptotic solution and performing a local asymptotic analysis near $t=\infty$, we get the decreasing eigenfunction $g=t^{-\frac{5}{8}} \exp \left\{-\frac{4}{7} 2^{\frac{1}{4}} t^{\frac{7}{4}}\right\}$, leading to the transcendental behavior

$$
\phi=2^{-\frac{3}{2}} t^{-\frac{5}{2}}+A g(t) \quad(t \rightarrow \infty),
$$


where the nonlinear eigenvalue $A$ can be found by solving $t \phi=K$ numerically [3], imposing the calculated asymptotic behaviour. The constant $B$ may be related to the nonlinear eigenvalue $A$ by matching the outer WKB correction (8) with the transcendental contribution found in the inner domain (13): $A=2^{-\frac{3}{8}} \sigma^{-\frac{13}{28}} B$. For the tip slope, we get

$$
\left.\frac{\mathrm{d} \eta_{s}}{\mathrm{~d} \xi}\right|_{\xi=0}=2^{\frac{11}{8}} \sigma^{-\frac{1}{28}} \operatorname{Im}(A) \exp \left\{\sigma^{-\frac{1}{2}} S(0)\right\}
$$

a result that shows that with isotropic capillary length there is no solution to the selection problem, as the righthand side of (14) is different from zero.

Let us now consider how the approach works when a potential flow is imposed externally, with the nondimensional flow velocity $\mathbf{U}$ tending to $-P_{f} \mathbf{e}_{y}$ for $y \rightarrow \infty$. We choose this irrotational frictionless flow, because for this case the exact solution of the problem without surface tension, i.e., the analog of the Ivantsov solution, is known [12]. Clearly, this is just a toy model, as it cannot be used to study viscosity effects. But it is a useful simple example in demonstrating our method, extensible to more realistic flow patterns without difficulties of principle. Introducing the stream function $\psi$ via $U_{x}=\partial_{y} \psi, U_{y}=-\partial_{x} \psi$, the bulk equation in the liquid region, now nonlinear, reads [12]:

$$
T_{\xi \xi}+T_{\eta \eta}=\psi_{\eta} T_{\xi}-\psi_{\xi} T_{\eta}+e^{-\frac{1}{2} P_{f}(\eta-1)^{2}} \psi_{\xi} .
$$

Here, the stream function $\psi$ is determined by $\psi_{\xi \xi}+\psi_{\eta \eta}=$ 0 with the boundary conditions $\lim _{\eta \rightarrow \infty}\left(\psi-\psi_{0}\right)=0$, where $\psi_{0}=P_{f}(\eta-1) \xi$ is the Ivantsov-like solution for the stream function obtained with $d_{0}=0$, and

$$
\psi_{\xi}=-P_{f}(\xi h)^{\prime}
$$

at the interface. The field equation in the solid region and the interface equations (3) remain unchanged. As before, we use (5) in the solid. Analogically to (4), we write

$$
\left(\partial_{\xi}+i \partial_{\eta}\right) \psi=0 .
$$

Equation (15), valid in the liquid region, does not factorize (not even asymptotically), but we may use Zauderer's asymptotic decomposition method [13] to achieve a similar reduction of order while keeping transcendentally small terms. In order not to overburden this first presentation, we linearize (15) about $\psi_{0}$. Again, this is by no means a necessary step. We have performed the full analysis without this linearization, which leads to the same equations (22) and (23) given below. We first rewrite equations (15) and (17) together with the boundary conditions (3) and (16), and using (5), as a first-order system:

$$
\mathbf{w}_{\xi}+A \mathbf{w}_{\eta}+\epsilon\left\{B \mathbf{w}+C \mathbf{w}_{\xi}\right\}=0
$$

with boundary condition

$$
\alpha \mathbf{w}+\beta \mathbf{w}_{\xi}=\mathbf{b},
$$

where $\mathbf{w}=\left(T_{\xi}, T_{\eta}, \psi\right)^{T}$ and

$$
\mathbf{b}=\left(\begin{array}{c}
h^{\prime}-\frac{\sigma}{2} \kappa^{\prime} \\
i \frac{\sigma}{2} \kappa^{\prime}-(\xi h)^{\prime} \\
-P_{f}(\xi h)^{\prime}
\end{array}\right), \quad A=\left(\begin{array}{ccc}
0 & 1 & 0 \\
-1 & 0 & 0 \\
0 & 0 & i
\end{array}\right)
$$

Using the Kronecker symbol $\delta_{i j}$, the remaining matrices may be written $B_{i j}=\delta_{i 1}\left[-P_{f} \xi \delta_{j 1}+P_{f}(\eta-1) \delta_{j 2}\right], C_{i j}=$ $-e^{-\frac{1}{2} P_{f}(\eta-1)^{2}} \delta_{i 1} \delta_{j 3}, \alpha_{i j}=\delta_{i j}\left(1-\delta_{i 3}\right), \beta_{i j}=\delta_{i j}-\alpha_{i j}$. $\epsilon$ has been inserted into (18) as a bookkeeping variable, to keep track of the order of Zauderer's decomposition scheme. The formal procedure would be to write $\xi=$ $-i+\epsilon X, \eta=1+\epsilon Y$ and express the equations in terms of $X$ and $Y$. In this presentation, we already decomposed the equations partially by writing Eq. (17). The parameter $\epsilon$ is assumed small as $\sigma \ll 1$. It is possible to determine the scaling exponent for $\epsilon$ in a more formal approach. $A$ has the following eigenvectors:

$$
\mathbf{r}_{11}=\left(\begin{array}{c}
-i \\
1 \\
0
\end{array}\right), \quad \mathbf{r}_{12}=\left(\begin{array}{c}
0 \\
0 \\
1
\end{array}\right), \quad \mathbf{r}_{2}=\left(\begin{array}{l}
i \\
1 \\
0
\end{array}\right)
$$

with eigenvalues $i, i,-i$, respectively. We look for a solution of the form

$$
\mathbf{w}=M \mathbf{r}_{11}+\psi \mathbf{r}_{12}+\epsilon N \mathbf{r}_{2},
$$

assuming $\epsilon N$ to be much smaller than $M$ and $\psi$. Expanding the resulting system of equations to first order in $\epsilon$, we obtain a set of decoupled first order equations. Setting $\epsilon=1$ and introducing characteristic coordinates $s=-i(\eta-1), \tau=\xi+i(\eta-1)$, we find $\psi=\psi(s, \tau)=-P_{f}[\tau h(\tau)]$, and using the boundary conditions at the interface, we arrive at the two equations

$$
\begin{gathered}
M=M(s, \tau)=\frac{i}{2} e^{\frac{P_{f}}{2}\left(s^{2}+\tau s\right)}\left\{[(1+i \tau) h(\tau)]^{\prime}\right. \\
\left.-2[\tau h(\tau)]^{\prime} \frac{1-e^{-\frac{P_{f}}{2} \tau s}}{\tau}\right\} \\
N=N(\bar{s}, \bar{\tau})=-\frac{P_{f}}{2} \bar{\tau} \int_{0}^{\bar{s}} \mathrm{~d} \bar{s}^{\prime} M\left(-\bar{s}^{\prime}, 2 \bar{s}^{\prime}+\bar{\tau}\right) \\
+i \frac{P_{f}}{2} \int_{0}^{\bar{s}} \mathrm{~d} \bar{s}^{\prime}\left[h\left(2 \bar{s}^{\prime}+\bar{\tau}\right)+\left(2 \bar{s}^{\prime}+\bar{\tau}\right) h^{\prime}\left(2 \bar{s}^{\prime}+\bar{\tau}\right)\right] e^{\frac{P_{f}}{2} \bar{s}^{\prime 2}} \\
+\frac{i}{2}\left\{\sigma \kappa^{\prime}(\bar{\tau})-[(1-i \bar{\tau}) h(\bar{\tau})]^{\prime}\right\}
\end{gathered}
$$

where $\bar{s}=i(\eta-1), \bar{\tau}=\xi-i(\eta-1)$. Next, we require $N$ to vanish for $\bar{s} \rightarrow i \infty$ while keeping $\bar{\tau}$ fixed. $\bar{\tau}$ may be interpreted as the continuation of the variable $\xi$ into the lower half of the complex plane. Thus we write $\xi$ instead 
of $\bar{\tau}$, and after some manipulations Eq. (23) yields

$$
\begin{aligned}
& (1-i \xi) h+\frac{P_{f}}{4} e^{\frac{1}{8} P_{f} \xi^{2}} \int^{\xi} \mathrm{d} \xi^{\prime} e^{-\frac{1}{8} P_{f} \xi^{\prime 2}}\left[\left(1-i \xi^{\prime}\right) \xi^{\prime} h\left(\xi^{\prime}\right)\right. \\
& \left.+\frac{P_{f}}{2} \int_{\xi^{\prime}}^{i \infty} \mathrm{d} \xi^{\prime \prime}\left(\xi^{\prime \prime}-\xi^{\prime}\right) \xi^{\prime \prime} h\left(\xi^{\prime \prime}\right) e^{\frac{P_{f}}{8}\left(\xi^{\prime \prime}-\xi^{\prime}\right)^{2}}\right]=\sigma \kappa \cdot(24)
\end{aligned}
$$

For $P_{f}=0$, Eq. (24) reduces to (11). The calculation of the transcendental mismatch in the presence of a potential flow parallels the procedure for the flowless case. We first calculate the WKB solution of (24):

$$
h=B_{1} e^{\frac{P_{f}}{16}}(1-i \xi)^{-\frac{5}{8}}(1+i \xi)^{-\frac{3}{8}} \exp \left\{\sigma^{-\frac{1}{2}} S(\xi)+\frac{P_{f}}{16} \xi^{2}\right\}
$$

with $S(\xi)$ given in Eq. (9). To obtain the inner equation, we follow Ben Amar [14] in her treatment of the flowless finite growth Péclet number case and use the same scaling as in the case without convection, i.e., Eq. (10) with $\alpha=\frac{2}{7}$. Equation (24) simplifies to

$$
t \phi+P_{1} \int^{t} \mathrm{~d} t^{\prime} t^{\prime} \phi\left(t^{\prime}\right)=K,
$$

with $K$ defined in Eq. (12) and $P_{1}=\sigma^{\frac{2}{7}} \frac{P_{f}}{4}$. To leading asymptotic order,

$$
P_{1} \int^{t} \mathrm{~d} t^{\prime} t^{\prime} \phi\left(t^{\prime}\right) \sim 2^{-\frac{3}{2}} t^{-\frac{3}{2}}, \quad(t \gg 1) .
$$

Linearizing equation (26) about (27) and performing a local asymptotic analysis near $t=\infty$ we obtain

$$
g=t^{-\frac{5}{8}} \exp \left\{\frac{P_{1}}{2} t\right\} \exp \left\{-\frac{4}{7} 2^{\frac{1}{4}} t^{\frac{7}{4}}\right\}
$$

leading to

$$
\phi=2^{-\frac{3}{2}} t^{-\frac{5}{2}}+A_{1}\left(P_{1}\right) g(t) \quad(t \rightarrow \infty),
$$

where $A_{1}\left(P_{1}\right)$ is a nonlinear eigenvalue and a function of $P_{1}$. Matching (25) and (29), we obtain the tip slope

$$
\left.\frac{\mathrm{d} \eta_{s}}{\mathrm{~d} \xi}\right|_{\xi=0}=2^{\frac{11}{8}} \sigma^{-\frac{1}{28}} \operatorname{Im}\left\{A_{1}\right\} \exp \left\{\frac{P_{f}}{16}\right\} \exp \left\{\sigma^{-\frac{1}{2}} S(0)\right\} \text {. }
$$

Obviously, the mismatch will remain nonzero except possibly for isolated values of $P_{1}$, a case that may be excluded by numerical evaluation of $A_{1}\left(P_{1}\right)$. Hence, there is no admissible solution.

To include surface tension anisotropy, we have to replace $\kappa$ in (24) with $R \kappa$, where

$$
R=1-\beta \cos (4 \theta)=1-\beta \cos \left[4 \arctan \left|\frac{\eta_{s} \eta_{s}^{\prime}-\xi}{\eta_{s}+\xi \eta_{s}^{\prime}}\right|\right] .
$$

Studying the vicinity of $\xi=-i$, we find

$$
t \phi+P_{1} \int^{t} \mathrm{~d} t^{\prime} t^{\prime} \phi\left(t^{\prime}\right)=K+b H K
$$

where $H=-2(\dot{\phi}-1)^{2} /\left[(\dot{\phi}+1)^{2}(\phi+t)^{2}\right]$ and $b=\beta \sigma^{-\frac{4}{7}}$. Demanding $\operatorname{Im}\left(A_{1}\right)$ to vanish, equation (32) constitutes an eigenvalue problem for the parameter $b$, to be solved numerically. Denoting the lowest eigenvalue by $b=b_{0}\left(P_{1}\right)$ the selection criterion reads

$$
\sigma=f\left(P_{1}\right) \beta^{7 / 4}, \quad f\left(P_{1}\right)=\left[b_{0}\left(P_{1}\right)\right]^{-7 / 4} .
$$

We will not elaborate on the details of this solution, as the purpose of this article is only a demonstration of the method. Further results for the particular physical system will be discussed in a forthcoming paper.

To summarize, we have introduced a method that combines matched asymptotics in the complex plane with the asymptotic decomposition of partial differential equations. This allows one to compute exponentially small terms beyond all orders (of asymptotic expansions) for partial differential equations on free boundaries, as we have shown for dendritic growth in a forced potential flow. In comparison with the BP approach [8], ours has several advantages, the most important being that it paves the way for a rigorous nonlinear asymptotic analysis, which in some cases [9] seems to be the only one that gives even qualitatively correct answers. We are not aware of any other method allowing this type of analysis with nonlinear field equations. Many problems to which the Kruskal-Segur method has been applied so far, including viscous fingering [15], the $\psi^{4}$ breather of particle physics [16], or capillary water waves [17], are actually free boundary problems. But for the method to be applicable, they first had to be recast as an ordinary differential equation or at least a single differential-integral equation. This is the reason, why only the simplest, in some cases even the most unrealistic, physical situations, have been studied by this method. Without the restriction, we anticipate a much wider class of problems to be tractable. Hence we expect our approach to open a new line of research into a plethora of hitherto untractable selection or solvability problems not only in crystal growth and similar problems of pattern forming interface dynamics (such as viscous fingering), but in a vast number of situations reducible to free boundary problems.

\section{REFERENCES}

[1] G. P. Ivantsov, Dokl. Akad. Naut. SSSR 58, 567 (1947).

[2] B. Caroli, C. Caroli, B. Roulet, and J. S. Langer, Phys. Rev. A 33, 442 (1986).

[3] M. Ben Amar and Y. Pomeau, Europhys. Lett. 2, 307 (1986).

[4] A. Barbieri, D. C. Hong, and J. S. Langer, Phys. Rev. A 35, 1802 (1987).

[5] E. A. Brener and V. I. Mel'nikov, Adv. Phys. 40, 53 (1991). 
[6] M. E.Glicksman and S. P. Marsh, The Dendrite. In Handbook of Crystal Growth, Vol. 1 (ed. D.T.J. Hurle), NorthHolland (1993), p. 1075.

[7] S. Tanveer, J. Fluid Mech. 409, 273 (2000).

[8] P. Bouissou and P. Pelcé, Phys. Rev. A 40, 6673 (1989).

[9] S. Tanveer, Proc. R. Soc. Lond. A 428, 511 (1990).

[10] M. D. Kruskal and H. Segur, Stud. Appl. Math. 85, 129 (1991).

[11] This linearization allows us to keep formulas less cumbersome. It should be avoided in a theory that is to become quantitatively accurate in the limit $d_{0} \rightarrow 0$. A presentation of the slightly more involved full calculation might obscure the essence of the approach and is postponed to a more technical extended paper. The result (6) remains exact in the full theory.

[12] M. Ben Amar, P. Bouissou, and P. Pelcé, J. Cryst. Growth 92, 97 (1988).

[13] E. Zauderer, Siam. Appl. Math. 35, 575 (1978).

[14] M. Ben Amar, Phys. Rev. A 41, 2080 (1990).

[15] R. Combescot, T. Dombre, V. Hakim, Y. Pomeau, and A. Pumir, Phys. Rev. Lett. 56, 2036 (1986).

[16] H. Segur and M. D. Kruskal, Phys. Rev. Lett. 58, 747 (1987).

[17] Y. Pomeau, A. Ramani, and B. Grammaticos, Physica D 31, 27 (1988). 